\title{
Competencia del ovocito bovino obtenido por ovum pick-up valorado mediante el azul brillante de cresilo
}

\author{
COMPETENCE OF THE BOVINE OOCYTE OBTAINED BY OVUM PICK-UP AS EVALUATED BY THE \\ BRIGHT CRESYL BLUE TEST
}

\author{
Luis Ayala G. ${ }^{1}$, Jorge Samaniego C..,3, Pedro Nieto E. ${ }^{1}$, Ramiro Rodas C. ${ }^{1}$, \\ Jorge Dután S. ${ }^{1}$, Guido Calle O. ${ }^{1}$, Yury Murillo A. ${ }^{1}$, Juan Vázquez M. ${ }^{1}$, \\ Daniel Argudo G. ${ }^{2}$, Fernando Perea G. ${ }^{1}$
}

\section{Resumen}

\begin{abstract}
El objetivo del presente estudio fue valorar la prueba del azul brillante de cresilo (BCB) como método indirecto para seleccionar ovocitos competentes para la producción in vitro de embriones (PIV). Los complejos cúmulos-ovocitos (COC) fueron obtenidos de dos vaquillas criollas sometidas a dos tratamientos: $\mathrm{T} 1=\mathrm{COC}$ recuperados por OPU (ovum pick-up) previa estimulación con FSH-LH; T2 = COC recuperados sin previa estimulación de la donante (testigo). Las dos vaquillas fueron alternadas en los dos tratamientos y se hicieron cinco repeticiones. Los $\mathrm{COC}$ recuperados fueron clasificados en tipos A, B, C y D. Luego se aplicó la prueba del BCB a cada uno de los tipos de COC para determinar si son $\mathrm{BCB}+$ o BCB-. T1 permitió recuperar 5.2 más COC que T2 $(\mathrm{p}<0.05)$. $\mathrm{Al}$ aplicar la prueba del $\mathrm{BCB}$ se determinó que todos los ovocitos tipo A de T1 y T2 fueron $\mathrm{BCB}+$; es decir, terminaron su crecimiento y se encontraban listos para iniciar el proceso de maduración in vitro; sin embargo, alrededor del 50\% de los COC tipo B, C y D de T1 y $\mathrm{T} 2$ fueron $\mathrm{BCB}+$. Se concluye que la selección de $\mathrm{COC}$ basado en las características morfológica es un método confiable únicamente para los de tipo $\mathrm{A}$, y tiene un $50 \%$ de error para los COC de tipo B, C y D y, por lo tanto, la aplicación de la prueba del BCB permite mejorar esta selección de forma no invasiva.
\end{abstract}

Palabras clave: COC bovinos; morfología ovocitaria; BCB; G6PDH

\footnotetext{
${ }^{1}$ Carrera de Medicina Veterinaria, Facultad de Ciencias Agropecuarias, Universidad de Cuenca, Ecuador

${ }^{2}$ Laboratorio de Biotecnología Animal, Facultad de Ciencias Agropecuarias, Universidad de Cuenca, Ecuador

${ }^{3}$ E-mail: jorgereivax@hotmail.com
}

Recibido: 17 de octubre de 2017

Aceptado para publicación: 16 de febrero de 2018 
The objective of the present study was to evaluate the bright blue cresyl (BCB) test as an indirect method to select competent oocytes for the in vitro production of embryos (IVP). The cumulus-oocyte complexes (COC) were obtained from two Creole heifers subjected to two treatments: $\mathrm{T} 1=\mathrm{COC}$ recovered by OPU (ovum pick-up) previous stimulation with FSH-LH; T2 = COC recovered from non-stimulated animals (control). The two heifers were alternated in the two treatments and five repetitions were done. Recovered COCs were classified into types A, B, C and D. Then the BCB test was applied to each of the $\mathrm{COC}$ types to determine if they were $\mathrm{BCB}+$ or BCB-. T1 allowed to recover 5.2 more $\mathrm{COC}$ than $\mathrm{T} 2(\mathrm{p}<0.05)$. When applying the $\mathrm{BCB}$ test, it was determined that all type A oocytes of $\mathrm{T} 1$ and $\mathrm{T} 2$ were $\mathrm{BCB}+$; that is, they finished their growth and were ready to start the process of in vitro maturation; however, about $50 \%$ of the type $\mathrm{B}, \mathrm{C}$ and $\mathrm{D}$ COCs of $\mathrm{T} 1$ and $\mathrm{T} 2$ were $\mathrm{BCB}+$. It is concluded that the selection of COC based on morphological characteristics is a reliable method only for type A and has a 50\% error for COC type B, C and D and, therefore, the application of the BCB test allows to improve this selection non-invasively.

Key words: bovine COC; oocyte morphology; BCB; G6PDH

\section{INTRODUCCIÓN}

La clasificación morfológica de los COC (complejos cumulus ovocitos), junto a la determinación del diámetro folicular y ovocitario, han sido los métodos más utilizados para la selección de gametas de óptima calidad previo a la producción in vitro de embriones (PIV) (Marchal et al., 2002; Anguita et al., 2007; Carrasco, 2012). En un principio, los COC aptos para PIV fueron seleccionados mediante criterios morfológicos que proporcionaban pautas razonables para identificar el potencial de fertilización, tomando en cuenta el espesor (número de capas), la compactación del cumulus, la homogeneidad y la tonalidad del ooplasma (Gordon, 2003); lo cual en el bovino ha demostrado una alta variabilidad al momento de evaluar el citoplasma debido a su poca o nula traslucidez, situación que no sucede en otras especies en las cuales la clasificación morfológica se correlaciona directamente con una buena capacidad de desarrollo (Goovaerts et al., 2010). Sin embargo, en los últimos años se ha comprobado que estos métodos son ineficientes, ya que apenas el $60 \%$ de los COC seleccionados alcanzan la fase de blastocisto posterior a la fecundación (Alm et al., 2005).

Ensayos realizados han demostrado que COC inmaduros sintetizan una variedad de proteínas durante su crecimiento, entre ellas la glucosa-6-fosfato-deshidrogenasa (G6PDH), la cual es sintetizada en la mitad de la primera fase (S) de crecimiento ovocitario y que disminuye conforme el ovocito termina su fase de crecimiento (Wassarman, 1988). Autores como Ericsson et al. (1993) y Roca et al. (1998) describieron que la tinción con azul brillante de cresilo (BCB) permite seleccionar de una manera no invasiva y perturbadora a los COC más homogéneos y competentes para la PIV; esto debido a que permite cuantificar la actividad de la enzima G6PDH, considerando a ovocitos que retienen la tinción como aquellos que terminaron su crecimiento $(\mathrm{BCB}+$; G6PDH inactiva) y ovocitos incoloros a aquellos que se encuentran en crecimiento (BCB-; G6PDH activa) (Pujol et al., 2004). 
Al ser considerada como una técnica relativamente nueva, existe poca documentación y mucha controversia en las diferentes especies de interés zootécnico; así, Mirshamsi et al. (2013) establecieron que un $54.3 \%$ de COC bovinos morfológicamente compactos eran $\mathrm{BCB}+\mathrm{y}$ podría ser utilizado para PIV; esto es corroborado por Pujol et al. (2004) quienes encontraron un porcentaje mayor de $\mathrm{COC} \mathrm{BCB}+$ en ovocitos de grado $1(78.6 \%)$ a comparación de COC grado $2(66.2 \%)$ y grado $3(51.1 \%)$. Sin embargo, en otras especies, Katska-Ksiazkiewicz et al. (2007) señalaron que no existe diferencia en la competencia de desarrollo entre ovocitos $\mathrm{BCB}+\mathrm{y}$ ovocitos clasificados como grado 1 (control) obtenidos de cabras. El presente estudio tuvo como objetivo utilizar la prueba del azul brillante de cresilo como método indirecto para seleccionar ovocitos de mayor competencia para la PIV.

\section{Materiales y Métodos}

\section{Colección de los COC}

Se utilizaron dos vaquillas criollas como donadoras de ovocitos, que fueron alternadas en dos tratamientos (T1: COC recuperados de vaquillas estimuladas; T2: $\mathrm{COC}$ recuperados de vaquillas no estimuladas [testigo]), cada uno con cinco repeticiones. Las hembras fueron sometidas al proceso de estimulación hormonal propuesto por Ruiz et al. (2013): Día 0: GnRH (Conceptal ${ }^{\circledR}$, Intervet), $0.2 \mathrm{mg}$ i.m.; Día 2: 500 UI FSHLH (Pluset ${ }^{\circledR}$, Laboratorios Calier, Barcelona); y 48 h después Ovum Pick-up (OPU).

Todos los folículos $\geq 4 \mathrm{~mm} \mathrm{y} \geq 8 \mathrm{~mm}$ fueron aspirados con el apoyo de un ecógrafo portátil (Prosound 2, Aloka, Japón) con un transductor sectorial de $5 \mathrm{MHz}$. Este último fue ensamblado en un soporte para sonda, que contenía la guía de punción conformada por una aguja $(1.2 \times 75 \mathrm{~mm})$ conectada a una bomba de vacío (BV-003D, WTA, Brasil) con una presión de aspiración de $70 \mathrm{mmHg}$. Los
COC recuperados fueron transportados en solución tampón fosfato salino (PBS) a $37{ }^{\circ} \mathrm{C}$ al laboratorio de biotecnología de la Facultad de Ciencias Agropecuarias de la Universidad de Cuenca, Ecuador,

\section{Clasificación}

Los COC fueron clasificados morfológicamente según los criterios descritos por Le Guienne (1999) en: A = ovocito de apariencia compacta con más de cuatro capas de células del cumulus, citoplasma granular uniforme $\mathrm{y}$ transparente; $\mathrm{B}=$ ovocito con 1 a 3 capas de células del cumulus que cubren la zona pelúcida, con citoplasma opaco, total o parcialmente homogéneo y finamente granulado; $\mathrm{C}=$ ovocitos totalmente denudados, y/o citoplasma con zonas oscuras irregulares y $\mathrm{D}=$ ovocitos deformados con células de la granulosa que cubren parcial o totalmente la zona pelúcida o completamente expandidos con cumulus disperso y descolorido.

\section{Prueba del Azul Brillante de Cresilo (BCB)}

Todos los ovocitos seleccionados como A, B, C y D fueron colocados en $26 \mu \mathrm{M}$ de BCB (B-5388, Sigma-Aldrich) diluido en PBS de Dulbecco durante 90 minutos a $38^{\circ} \mathrm{C}$ y $\mathrm{CO}_{2}$ al $5 \%$. Luego, cada grupo de ovocitos fue lavado tres veces en una solución atemperada de PBS y examinado bajo estereoscopía para determinar si habían terminado su crecimiento (BCB+) o no (BCB-) (Pujol et al., 2004).

\section{Diseño Experimental}

Como criterio de inclusión se consideraron ovocitos provenientes de folículos con diámetro entre 4 y $8 \mathrm{~mm}$ y como criterio de exclusión a ovocitos contaminados con bacterias durante el cultivo. La punción folicular se realizó una vez por semana entre diciembre de 2016 y marzo de 2017. Todas las punciones fueron realizadas por el mismo Médico Veterinario. 
Se utilizó un modelo lineal general a través del PROC GLM en el SAS v. 9.3 (SAS, 2013). Las diferencias entre medias se compararon mediante la prueba de TukeyKramer.

\section{Resultados y Discusión}

El Cuadro 1 muestra la relación entre el número de folículos visualizados por ecografía y el número de COC recuperados por OPU. Como era de esperarse, el tratamiento 1 (con estimulación) permitió recuperar 5.2 más COC que el grupo testigo (sin estimulación) $(p<0.05)$. La tasa de recuperación en T1 fue $17.1 \%$ superior a T2 $(\mathrm{p}<0.05)$. El porcentaje de recuperación denota la eficiencia alcanzada en la técnica del OPU, en tanto que la diferencia entre tratamientos podría ser explicada por el protocolo de estimulación utilizado, que permite obtener folículos más homogéneos que facilitan la punción ecoguiada (Pieterse et al., 1988; De Roover et al., 2005; Torres et al., 2008). Asimismo, los resultados obtenidos concuerdan con De Roover et al. (2005), quienes señalan que un animal estimulado hormonalmente antes del OPU aumenta significativamente el número de folículos visualizados y $\mathrm{COC}$ recuperados con respecto a aquellos que no recibieron estimulación.

En el Cuadro 2 se puede observar que del total de COC recuperados $(9.0 \pm 0.63)$ de animales estimulados hormonalmente, el $26.7 \%$ corresponden al tipo A, $34.4 \%$ al B, $21.1 \%$ al $\mathrm{C}$ y $17.8 \%$ al $\mathrm{D}$, mientras que para $\mathrm{T} 2$, el $13.8 \%$ fueron clasificados como A, $25.0 \%$ como B, $47.4 \%$ como y $13.8 \%$ como $\mathrm{D}$, siendo las frecuencias en los tipos A, B y D significativamente diferentes $(p<0.05)$.

Diversos autores consideran a ovocitos de las categorías A y B como aptos para PIV y a $C$ y D como no aptos para esta biotecnología (Humblot et al., 2005; Ireland et al., 2007 ; Katska-Ksiazkiewicz et al.,
2007). Es así, que se puede decir que en el $61.1 \%$ de los ovocitos en $\mathrm{T} 1$ serían considerados como aptos y en solo el $38.8 \%$ en T2 serían aptos. Esto es corroborado por Restrepo et al. (2011) quienes describen que este comportamiento se debe al efecto de la FSH y LH, que no solo permite el reclutamiento y crecimiento de un mayor número de folículos (efecto FSH) sino que, además, ejerce un efecto favorable sobre la calidad de los COC recuperados (efecto LH). Blondin et al. (2012) señalaron que ovocitos provenientes de hembras no estimuladas hormonalmente tuvieron menor competencia que ovocitos de hembras estimuladas, lo cual concuerda con los resultados de esta investigación.

Al realizar la prueba del $\mathrm{BCB}$, se determinó que el $100 \%$ de los ovocitos de tipo $\mathrm{A}$, tanto de $\mathrm{T} 1$ como de $\mathrm{T} 2$, son $\mathrm{BCB}+$; es decir, terminaron su crecimiento y pueden continuar con su proceso de maduración. Este resultado es mayor al obtenido por Pujol et al. (2004) quienes determinaron un $78.6 \%$ de $\mathrm{BCB}+$ en ovocitos de tipo 1. Esta diferencia puede ser explicada por la procedencia de los ovocitos que en su investigación fueron de ovarios de matadero. Rodríguez et al. (2002) y Alm et al. (2005) ratifican que la prueba de BCB se ve influenciada directamente por el grado morfológico de los ovocitos.

Alrededor del 55\% de los ovocitos de tipo $\mathrm{B}$ son $\mathrm{BCB}+$, lo que permite argumentar de que no todos los ovocitos de tipo B están listos para continuar con el proceso de maduración. Este comportamiento fue observado por Pujol et al. (2004), quienes demostraron que un $34 \%$ de los ovocitos seleccionados para PIV, según criterios morfológicos (grados 1 al 3), no han terminado su crecimiento; es decir, eran BCB-. Así mismo, se encontró un porcentaje mayor de $\mathrm{BCB}+$ en $\mathrm{COC}$ de grado 1 en comparación con $\mathrm{COC}$ grado 2, e igual comportamiento fue observado entre $\mathrm{COC}$ de grado 2 y 3. 
Cuadro 1. Número de folículos visualizados (n [promedio \pm error estándar]) mediante ultrasonido y COC recuperados (\%) por ovum pick-up ${ }^{1}$

\begin{tabular}{lccc}
\hline & $\begin{array}{c}\text { Folículos } \\
\text { visualizados }\end{array}$ & $\begin{array}{c}\text { COC } \\
\text { recuperados }\end{array}$ & Recuperación \\
\hline $\begin{array}{l}\text { T1, COC recuperados de vaquillas } \\
\text { estimuladas }\end{array}$ & $112\left(11.2 \pm 0.69^{\mathrm{a}}\right)$ & $90\left(9.0 \pm 0.63^{\mathrm{a}}\right)$ & $80.4^{\mathrm{a}}$ \\
$\begin{array}{l}\text { T2, COC recuperados de vaquillas } \\
\text { no estimuladas }\end{array}$ & $60\left(6.0 \pm 0.89^{\mathrm{b}}\right)$ & $23\left(3.8 \pm 0.57^{\mathrm{b}}\right)$ & $63.3^{\mathrm{b}}$ \\
\hline Total & $172(17.2)$ & $113(12.8)$ & 65.7 \\
\hline $\begin{array}{l}{ }^{1} \text { Cinco colecciones por vaquilla por tratamiento } \\
\mathrm{a}, \mathrm{b} \text { Valores con letras diferentes en la misma columna indican diferencia estadística }(\mathrm{p}<0.05)\end{array}$
\end{tabular}

Cuadro 2. COC (promedio \pm error estándar, [porcentaje]) clasificados en base a su morfología $(\mathrm{A}, \mathrm{B}, \mathrm{C}, \mathrm{D})^{1}$ por tratamiento y su actividad al azul brillante de cresilo (BCB)

\begin{tabular}{ccccc}
\hline & $\mathrm{A}$ & $\mathrm{B}$ & $\mathrm{C}$ & $\mathrm{D}$ \\
\hline $\mathrm{COC}$ & & & & \\
$\mathrm{T} 1$ & $2.4 \pm 0.38^{\mathrm{a}}(26.7)$ & $3.1 \pm 0.55^{\mathrm{a}}(34.4)$ & $1.9 \pm 0.44(21.1)$ & $1.6 \pm 0.30^{\mathrm{a}}(17.8)$ \\
$\mathrm{T} 2$ & $0.5 \pm 0.35^{\mathrm{b}}(13.8)$ & $0.9 \pm 0.50^{\mathrm{b}}(25.0)$ & $1.8 \pm 0.40(47.4)$ & $0.5 \pm 0.27^{\mathrm{b}}(13.8)$ \\
$B C B+$ & & & & \\
T1 & $2.4 \pm 0.38^{\mathrm{a}}(100)$ & $1.7 \pm 0.37^{\mathrm{a}}(54.8)$ & $1.3 \pm 0.36(68.4)$ & $0.9 \pm 0.26(56.3)$ \\
$\mathrm{T} 2$ & $0.5 \pm 0.35^{\mathrm{b}}(100)$ & $0.5 \pm 0.34^{\mathrm{b}}(55.5)$ & $1.0 \pm 0.33(55.5)$ & $0.3 \pm 0.24(60.0)$ \\
$B C B-$ & & & & \\
T1 & $0.0 \pm 0.00(0)$ & $1.4 \pm 0.54(45.2)$ & $0.6 \pm 0.34(31.6)$ & $0.7 \pm 0.23(43.7)$ \\
T2 & $0.0 \pm 0.00(0)$ & $0.3 \pm 0.49(44.5)$ & $0.9 \pm 0.31(44.5)$ & $0.1 \pm 0.21(40.0)$ \\
\hline
\end{tabular}

T1: COC recuperados de vaquillas estimuladas; $\mathrm{T} 2, \mathrm{COC}$ recuperados de vaquillas no estimuladas

${ }^{1}$ Le Guienne (1999)

$a, b$ Letras diferentes dentro de columnas indican grupos diferentes $(p<0.05)$

Por otra parte, al analizar los ovocitos de tipo $\mathrm{C}$ y $\mathrm{D}$ en conjunto no se observan diferencias entre tratamientos, pero se determinó que alrededor del $60 \%$ habían culminado su crecimiento $(\mathrm{BCB}+)$. En cuanto a los $\mathrm{COC}$ de tipo $\mathrm{C}$ (COC denudados), un alto porcentaje (aprox. 61\%) fue $\mathrm{BCB}+$. Este comportamiento pudo ser efecto indirecto de factores técnicos relacionados con la geome- tría de la aguja (diámetro, longitud, afilado, ángulo, etc.) o la presión de aspiración que produjo pérdida de las células del cúmulo (Ruiz, 2010; Boni, 2012). En cuanto a los ovocitos de tipo $\mathrm{D}$, su comportamiento $\mathrm{BCB}+$ se debe a que morfológicamente son maduros; es decir, terminaron su crecimiento y, es por eso que, diversos investigadores excluyen a estos ovocitos de la PIV, al ser consi- 
derados como no aptos para esta biotecnología (Pujol et al., 2004; Alm et al., 2005; Chaubal et al., 2006; Ireland et al., 2007 ; Ding et al., 2008). Por otra parte, se observa que alrededor del $50 \%$ de los ovocitos tipo D son BCB-, y se considera que estos son ovocitos producto de folículos en fase de atresia.

\section{Conclusiones}

La selección de COC apoyada en la prueba no invasiva del azul brillante de cresilo (BCB) permite mejorar la selección de gametos que tradicionalmente se fundamenta en la morfología y características del citoplasma del ovocito.

\section{Literatura Citada}

1. Alm H, Torner H, Löhrke B, Viergutz T, Ghoneim IM, Kanitz W. 2005. Bovine blastocyst development rate in vitro is influenced by selection of oocytes by brillant cresyl blue staining before IVM as indicator for glucose-6phosphate dehydrogenase activity. Theriogenology 63: 2194-2205. doi: 10.1016/j.theriogenology.2004.09.050

2. Anguita B, Jimenez-Marcedo A, Izquierdo D, Mogas T, Paramio M. 2007. Effect of oocyte diameter on meiotic competence, embryo development, p34 (cdc2) expression and MPF activity in prepubertal goat oocytes. Theriogenology 67: 526-536. doi: 10.1016/j.theriogenology.2006.09.003

3. Blondin P, Vigneault $C$, Nivet $A L$, Sirard MA. 2012. Improving oocyte quality in cows and heifers - What have we learned so far? Anim Reprod 9: 281289.

4. Boni R. 2012. Ovum pick-up in cattle: a $25 \mathrm{yr}$ retrospective analysis. Anim Reprod 9: 362-369.
5. Carrasco R. 2012. Uso de azul brillante de cresilo en la seleccion de ovocitos bovinos: Implicancias en la maduracion nuclear y citoplasmatica in vitro. Tesis de Médico Veterinario: Vadivia, Chile: Univ. Austral de Chile. 22 p.

6. Chaubal SA, Molina JA, Ohlrichs CL, Ferre LB, Faber DC, Bols PE., Riesen JW, Yang, $X$. 2006. Comparison of different transvaginal ovum pick-up protocols to optimise oocyte retrieval and embryo production over a 10 -week period in cows. Theriogenology 65: 16311648. doi: 10.1016/j.theriogenology.2005.07.020

7. Ding L, Tian H, Wang J, Chen J, Sha H, Chen J, Cheng G 2008. Different intervals of ovum pick-up affect the competence of oocytes to support the preimplantation development of cloned bovine embryos. Mol Reprod Dev 75: 1710-1715. doi: 10.1002/mrd.20922

8. Ericsson SA, Boice ML, Funahashi H, Day BN. 1993. Assessment of porcine oocytes using brilliant cresyl blue. Theriogenology 39: 214. doi: 10.1016/ 0093-691X(93)90069-H

9. Goovaerts I, Leroy J, Jorssen E, Bols $P$. 2010. Non invasive bovine oocyte quality assessment: possibilities of a single oocyte culture. Theriogenology 74 : 1509-1520. doi: 10.1016/j.theriogenology.2010.06.022

10. Gordon I. 2003. Laboratory production of cattle embryos. $2^{\text {nd }}$ ed. Cambridge: CAB International. $592 \mathrm{p}$.

11. Humblot P, Holm P, Lonergan P, Wrenzycki C, Leguarré AS, Joly CG, Fermann D, et al. 2005. Effect of stage of follicular growth during superovulation on developmental competence of bovine oocytes. Theriogenology 63: 1149-1166. doi: 10.1016/j.theriogenology.2004.06.002

12. Ireland JJ, Ward F, Jimenez-Krassel F, Ireland J, Smith GW, Lonergan P, Evans $\boldsymbol{A C}$. 2007. Follicle numbers are highly repeatable within individual animals but are inversely correlated with 
FSH concentrations and the proportion of good-quality embryos afterovarian stimulation in cattle. Huma Reprod 22: 1687-1695. doi: 10.1093/humrep/dem071

13. Katska-Ksiazkiewicz L, Opiela J, Rynska B. 2007. Effects of oocyte quality, semen donor and embryo coculture system on the efficiency of blastocyst production in goats. Theriogenology 68: 736-744. doi: 10.1016/j.theriogenology.2007.06.016

14. Le Guienne M. 1999. Atlas of the bovine oocyte. AETE Newsletter $10: 6-8$.

15. Marchal $R$, Vigneron $C$, Perreau $C$, Bali-Papp A, Mermillod P. 2002. Effect of follicular size on meiotic and developmental competence of porcine oocytes. Theriogenology 57: 1523-1532. doi: 10.1016/S0093-691X(02)00655-6

16. Mirshamsi SM, Karamishabankareh H, Ahmadi-Hamedani M, Soltani L, Hajarian H, Abdolmohammadi AR. 2013. Combination of oocyte and zygote selection by brilliant cresyl blue (BCB) test enhanced prediction of developmental potential to the blastocyst in cattle. Anim Reprod Sci 136: 246-251. doi: 10.1016/j.anireprosci.2012.11.002

17. Pieterse MC, Kappen KA, Kruip TA, Taverne MA. 1988. Aspiration of bovine oocytes during transvaginal ultrasound scanning of the ovaries. Theriogenology 30: 751-762. doi: 10.1016/0093-691X(88)90310-X

18. Pujol M, López-Bejar M, Paramio MT. 2004. Developmental competence of heifer oocytes selected using the brilliant cresyl blue (BCB) test. Theriogenology 61: 735-744. doi:10.1016/S0093691X(03)00250-4

19. Restrepo G, Gómez J, Vásquez N. 2011. Evaluación de la superestimulación ovárica y la calidad morfológica de oocitos bovinos obtenidos por aspiración folicular. Rev Politécnica 7(13): 16-21.
20. Roca J, Martínez E, Vázquez JM, Lucas X. 1998. Selection of immature pig oocytes for homologous in vitro penetration assays with the brilliant cresyl blue test. Reprod Fert Dev 10: 479-485. doi: 10.1071 / RD98060

21. Rodriguez-González E, López-Béjar M, Velilla E, Paramio MT. 2002. Selection of prepubertal goat oocytes using the brilliant cresyl blue test. Theriogenlogy 57: 1397-1409. doi: 10.1016/S0093-691X (02) 00645-3

22. De Roover R, Bols P, Genicot G, Hanzen C. 2005. Characterisation of low, medium and high responders following FSH stimulation prior to ultrasound-guided transvaginal oocyte retrieval in cows. Theriogenology 63: 1902-1913. doi: 10.1016/j.theriogenology.2004.08.011

23. Ruiz S. 2010. Ovum pick up (OPU) en bovinos: aplicaciones en biotecnología de la reproducción. Cría y Salud 31: 58-64.

24. Ruiz S, Romero J, Astiz S, Peinado B, Almela L, Poto A. 2013. Aplication of reproductive biotechnology for the recovery of endangered breeds: birth of the first calf of Murciana-Levantina bovine breed derived by OPU, in vitro production and embryo vitrification. Reprod Domest Anim 48: e81-e84. doi: 10.1111/rda.12179

25. SAS. 2013. SAS Users guide. Cary, N.C, USA.

26. Torres J, de FA Pires $M$, de Sá, WF, de M Ferreira, A, Viana $J H$, Camargo LS., Ramos AA, et al. 2008. Effect of maternal heat-stress on follicular growth and oocyte competence in Bos indicus cattle. Theriogenology 69: 155-166. doi: 10.1016/j.theriogenology.2007.06.023

27. Wassarman M. 1988. The mammalian ovum. En: Knobil E, Neil D (eds). The physiology of reproduction. New York: Raven Press. p. 69-102. 